\title{
RECENT PROGRESS ON GRBS WITH SWIFT
}

\author{
N. Gehrels ${ }^{1}$ and J.K. Cannizzo ${ }^{2}$
}

\begin{abstract}
We are in an exciting period of discovery for gamma-ray

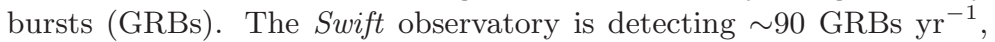
providing arcsecond localizations and sensitive observations of the prompt and afterglow emission. In addition, rapid-response telescopes on the ground are providing new capabilities to study optical and radio emissions. The combined data set is enabling great advances in our understanding of GRBs including afterglow physics, short burst origin, and the GRB-supernova connection.
\end{abstract}

\section{Introduction}

GRBs are the most luminous explosions in the universe and are thought to be the birth cries of black holes. They are a product of the space age, discovered (Klebesadel et al. 1973) by Vela and observed by satellites for 40 years. Despite impressive advances over the past three decades, the study of bursts remains highly dependent on the capabilities of the observatories which carried out the measurements. The era of the Compton Gamma Ray Observatory (CGRO) led to the discovery of more than 2600 bursts in just 9 yr. Analyses of these data produced the key result that GRBs are isotropic on the sky and occur at a frequency of roughly two per day all sky (Meegan et al. 1991). The hint from earlier instruments was confirmed that GRBs come in two distinct classes of short and long bursts, with distributions crossing at $\sim 2$ s duration (Kouveliotou et al. 1993). The BeppoSAX mission made the critical discovery of X-ray afterglows of long bursts (Costa et al. 1997). With the accompanying discoveries by ground-based telescopes of optical (van Paradijs et al. 1997) and radio (Frail et al. 1997) afterglows, long GRBs were found to emanate from star forming regions in host galaxies at a typical distance of $z=1$. BeppoSAX and the later HETE-2 mission also found evidence of associations of GRBs with Type Ic SNe. This supported

\footnotetext{
1 Astroparticle Physics Division, NASA/Goddard Space Flight Center, Greenbelt, MD 20771, USA

2 CRESST/Joint Center for Astrophysics, Univ. of Maryland, Baltimore County, Baltimore, MD 21250, USA
} 


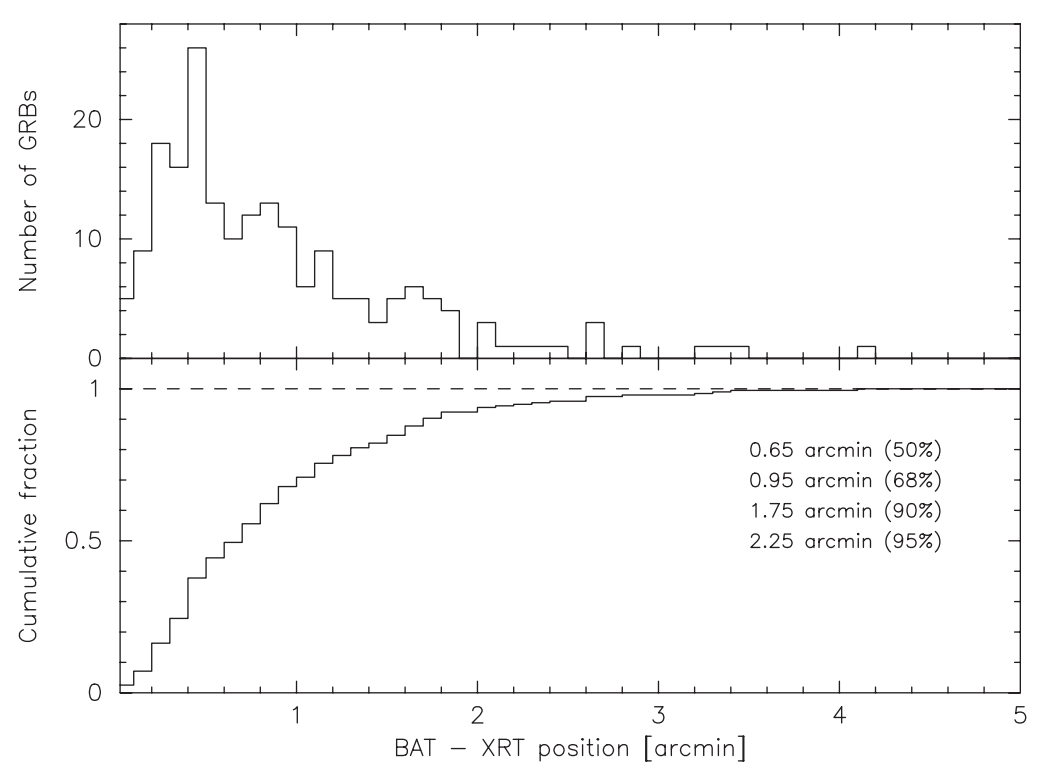

Fig. 1. Histogram (top) and the cumulative fraction (bottom) of the angular difference between the BAT ground position and the XRT position; $68 \%$ and $90 \%$ of BAT ground positions are within 0.95 and 1.75 arcmin from the XRT position, respectively (Sakamoto et al. 2008).

the growing evidence that long GRBs are caused by "collapsars" where the central core of a massive star collapses to a black hole (MacFadyen \& Woosley 1999).

\section{Swift GRBs}

Swift (Gehrels et al. 2004) is a dedicated GRB observatory that is now measuring many properties of the prompt and afterglow radiation. It carries a wide-field Burst Alert Telescope (BAT) (Barthelmy et al. 2005a) that detects GRBs and positions them to arcmin accuracy, and the narrow-field X-Ray Telescope (XRT) (Burrows et al. 2005) and UV-Optical Telescope (UVOT) (Roming et al. 2005) that observe their afterglows and determine positions to arcsec accuracy, all within $\sim 100 \mathrm{~s}$. The BAT detects the bursts in the $15-150 \mathrm{keV}$ band and determines a position accurate to within $\sim 2$ arcminutes (Fig. 1) on-board within $12 \mathrm{~s}$. The position is provided to the spacecraft which is then repointed to the burst location in less than 2 minutes to allow XRT (Fig. 2) and UVOT observations of the afterglow. Alert data from all three instruments is sent to the ground via NASA's TDRSS relay satellite. The full data set is stored and dumped to the Italian Space Agency's equatorial Malindi Ground Station.

The Swift mission was built by an international team from the US, UK, and Italy, with contributions also from Germany and Japan. After five years of 


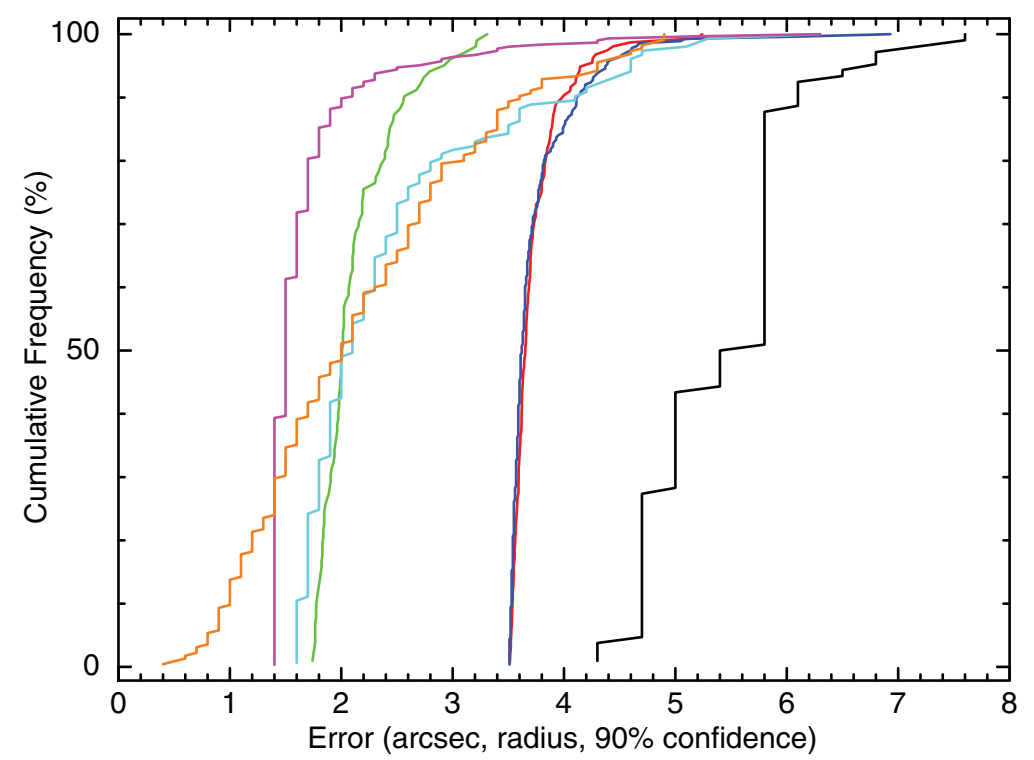

Fig. 2. The cumulative frequency of the $90 \%$ confidence error radius for XRT GRB positions determined using different techniques (Evans et al. 2009). We highlight two curves, the green curve which gives the on-board corrected positions, and the black curve which gives the on-board raw positions.

development it was launched form Kennedy Space Center on 20 November 2004. Full normal operations commenced on 5 April 2005.

BAT has detected $\sim 720$ GRBs in $8 \mathrm{yr}$, a rate of $\lambda=90 \mathrm{yr}^{-1}$. The cumulative distribution of time intervals between successive GRBs follows $e^{-\lambda t}$ (Fig. 3). Approximately $90 \%$ of the BAT-detected GRBs have repointings within 5 minutes (the remaining 10\% have spacecraft constraints that prevent rapid slewing). Of those, virtually all bursts observed promptly have detected X-ray afterglow. Already, $80 \%$ of the known X-ray afterglows are from Swift. The fraction of rapidpointing GRBs that have UVOT detection is $\sim 30 \%$. Combined with ground-based optical observations, about $60 \%$ of Swift GRBs have optical afterglow detection. To date there are a total of 273 redshift determinations, of which 41 are pre-Swift bursts (Fig. 4). There are 225 redshifts for Swift GRBs.

GRBs are incredibly bright. A typical galaxy at a redshift of only $z=3$ is fainter than $m \simeq 27$ in the optical, whereas GRBs can be in the range $m \simeq 15-20$. The brightest was a naked eye object. Table 1 presents optical data for the highest redshift GRBs observed to date, where the look-back time $t_{L B}$ is given in column 2 . Multiwavelength observations of the current record holder, GRB 090429B (at $z \simeq$ 9.4 ), are providing information about the universe at a time when it was only about $4 \%$ of its current age, and shed light on the process of reionization in the early universe (Cucchiara et al. 2011). 


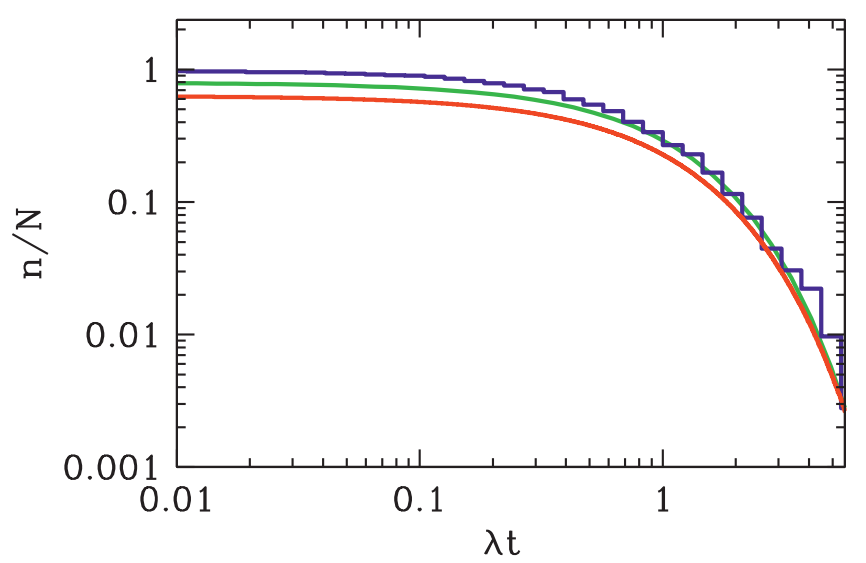

Fig. 3. The cumulative frequency distribution function for the intervals of time between all 720 Swift GRBs observed to date (counting from the largest values downward), shown in blue. For comparison the green curve shows $e^{-\lambda t}$, where $\lambda=90 \mathrm{yr}^{-1}$, and the red curve shows the results from a Monte Carlo simulation with $N=10^{6}$. For ease of viewing the green and red curves have been shifted downward 0.1 and 0.2 dex, respectively. The value $\lambda t=1$ along the $x$-axis corresponds to the mean interval between Swift GRBs of $4.06 \mathrm{~d}$.

Table 1. High $z$ GRBs.

\begin{tabular}{lccccc}
\hline \hline$z$ & $t_{\mathrm{LB}}(\mathrm{Gyr})$ & GRB & Brightness & & \\
\hline \hline 9.4 & 13.1 & $090429 \mathrm{~B}$ & $K=19$ & $@$ & $3 \mathrm{hr}$ \\
8.2 & 13.0 & 090423 & $K=20$ & $@$ & $20 \mathrm{~min}$ \\
$\sim 8$ & 13.0 & 120923 & & & \\
6.7 & 12.8 & 080813 & $K=19$ & $@$ & $10 \mathrm{~min}$ \\
6.3 & 12.8 & 050904 & $J=18$ & $@$ & $3 \mathrm{hr}$ \\
5.6 & 12.6 & 060927 & $I=16$ & $@$ & $2 \mathrm{~min}$ \\
5.3 & 12.6 & 050814 & $K=18$ & $@$ & $23 \mathrm{hr}$ \\
5.11 & 12.5 & 060522 & $R=21$ & $@$ & $1.5 \mathrm{hr}$ \\
\hline \hline
\end{tabular}

\subsection{Short GRBs}

At Swift's launch, the greatest mystery in GRB astronomy was the nature of shortduration, hard-spectrum bursts (Fig. 5). Although more than 50 long GRBs had afterglow detections, no afterglow had been found for any short burst. In May 2005 (GRB 050509B), Swift provided the first short GRB X-ray afterglow localization (Gehrels et al. 2005). This burst plus the HETE-2 GRB 050709 and Swift GRB 050724 led to a breakthrough in our understanding of short bursts (Gehrels et al. 2005; Bloom et al. 2006; Fox et al. 2005; Villasenor et al. 2005; Hjorth et al. 2005; Barthelmy et al. 2005b; Berger et al. 2005). There are now 68 localizations 

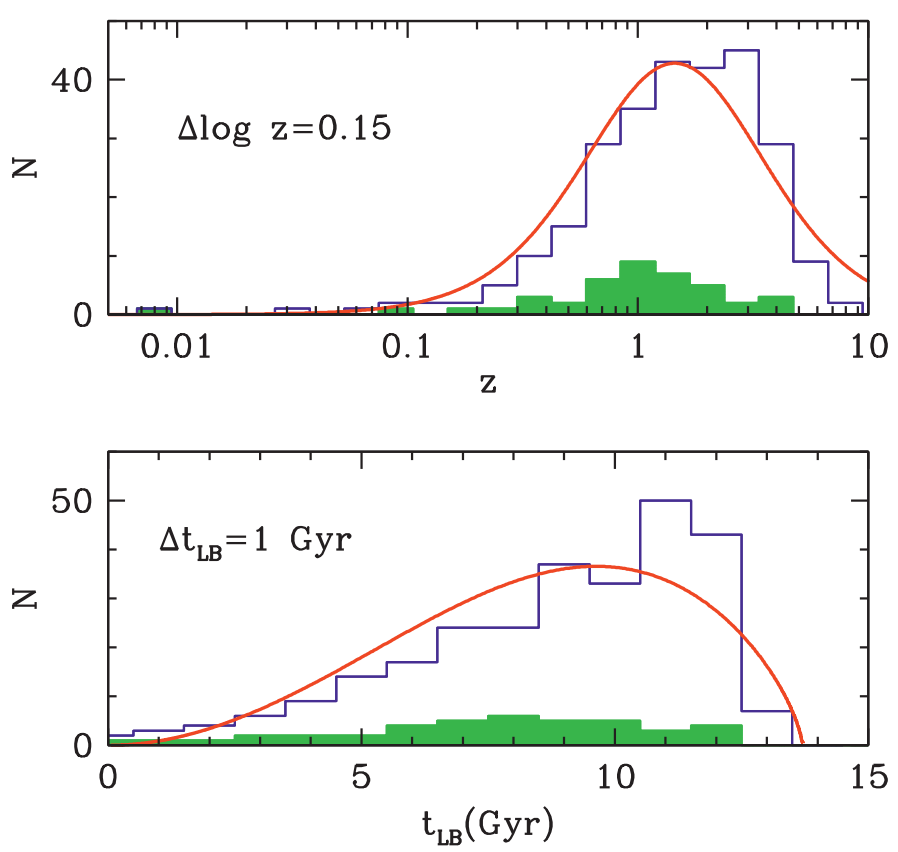

Fig. 4. The distribution of redshifts for all GRBs to date $(N=273$, shown in blue). The green distribution indicates the pre-Swift values $(N=41)$, the red line indicates the evolution of a comoving volume element, $(d V / d z)(1+z)^{-1}$, and the lower panel shows the distribution plotted in look-back time $t_{\mathrm{LB}}$. The normalizations on the red lines are calculated so as to minimize the sum of the squares of the differences with the histogram values in each bin. The factor $(1+z)^{-1}$ multiplying the comoving volume element is necessary to account for cosmological time dilation, given that the GRB rate has units volume ${ }^{-1}$ time $^{-1}$.

for short GRBs, mainly from Swift. Most of these have XRT detections, and about one third have host identifications or redshifts.

In stark contrast to long bursts, the evidence starting from the first accurately localized short bursts is that they can originate from regions with low star formation rate. GRB 050509B and 050724 were from elliptical galaxies with low current star formation rates, while GRB 050709 occurred in a region of a star forming galaxy with no nebulosity or evidence of recent star formation activity in that location. Recent HST observations of locations of short GRBs in their hosts reveal that short bursts trace the light distribution of their hosts while long bursts are concentrated in the brightest regions (Fong et al. 2010). Short GRBs are also different from long GRBs in that accompanying supernovae are not detected for nearby events (Bloom et al. 2006; Fox et al. 2005; Hjorth et al. 2005). Taken together, these results support the interpretation that short bursts are associated with an old stellar population, and may arise from mergers of compact binaries [i.e., double neutron star or neutron star - black hole (NS-BH) binaries]. 


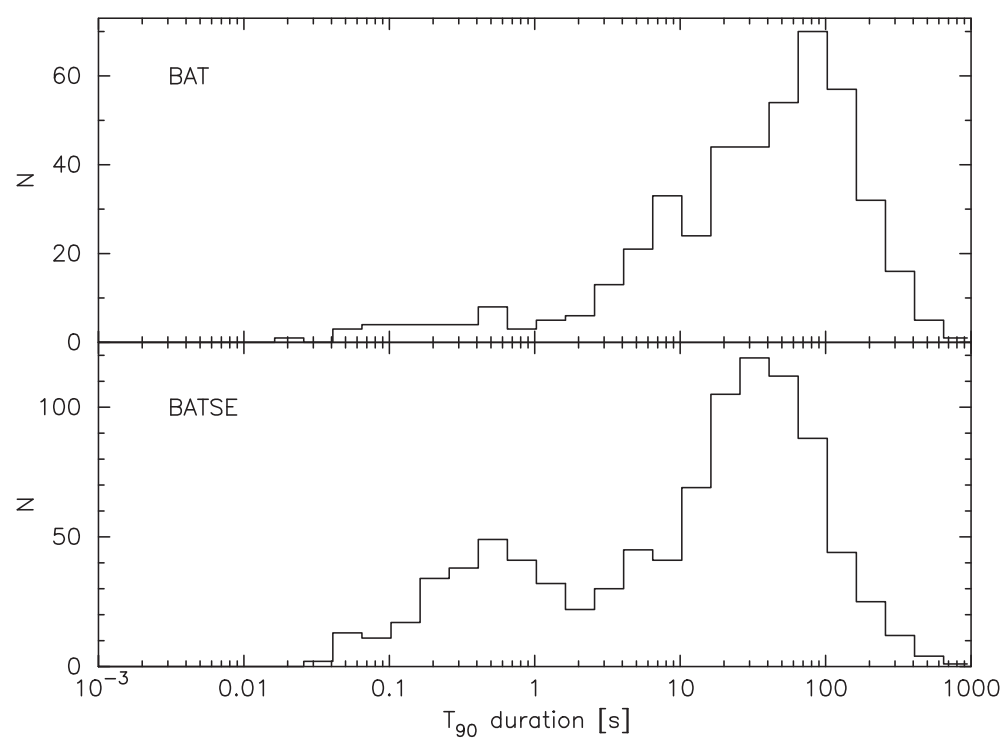

Fig. 5. $T_{90}$ distribution of BAT GRBs from the mask-weighted light curves in the $15-350 \mathrm{keV}$ band and the corresponding distribution for CGRO/BATSE GRBs from the light curves in the $50-350 \mathrm{keV}$ band (Sakamoto et al. 2011). Short GRBs constitute about $9 \%$ of the total number for BAT GRBs, and about $25 \%$ of the total number for BATSE GRBs. This difference is due to the narrower BAT energy band.

\subsection{Interesting case of the hostless GRB 070125}

There was not an obvious host galaxy for GRB 070125. Deep ground-based imaging reveals no host to $R>25.4 \mathrm{mag}$. Cenko et al. (2008) present an analysis of spectroscopic data which reveals only weak $\mathrm{Mg}$ II lines indicative of halo gas. In the field are two blue galaxies offset by $\gtrsim 27 \mathrm{kpc}$ at $z=1.55$. If there is an association with one of them, it would imply a velocity $\sim 10^{4} \mathrm{~km} \mathrm{~s}^{-1}$ over a $\sim 20 \mathrm{Myr}$ lifetime of the massive progenitor. The only known way of achieving this would have been a prior close interaction with a massive $\mathrm{BH}$. However, this interpretation was muddied by Chandra et al. (2008), who inferred a dense environment, based on bright, self-absorbed radio afterglow. They proposed a scenario in which the high density material lies close to the explosion site, and the lower density material further away. They note GRB 070125 was one of the brightest GRBs ever detected, with an isotropic release of $10^{54} \mathrm{erg}$ (by comparison, $M_{\odot} c^{2} \simeq 2 \times 10^{54} \mathrm{erg}$ ). The prompt emission from GRB 070125 was also seen by Suzaku/WAM (Onda et al. 2010).

\subsection{GRBs and supernovae}

On 18 February 2006 Swift detected the remarkable burst GRB 060218 that provided considerable new information on the connection between SNe and GRBs. 


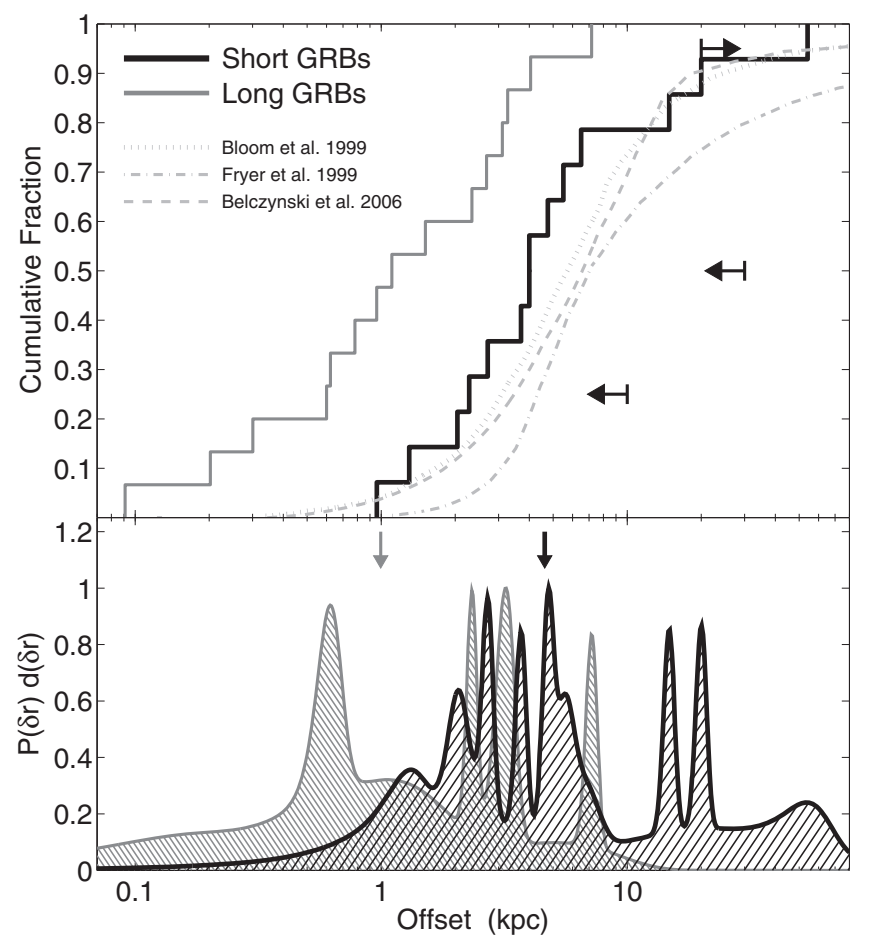

Fig. 6. Projected physical offsets within their host galaxies based on $H S T$ observations for short GRBs (black) and long GRBs (gray), from Fong et al. (2010). Data from long GRBs taken from (Bloom et al. 2002). Top panel shows the cumulative distributions; bottom panel shows the differential distributions. Arrows in the bottom panel mark the median value for each distribution. The median value for short GRBs, $\approx 5 \mathrm{kpc}$, is about 5 times larger than for long GRBs. Arrows in the top panel exhibit the strongest constraints on the offset distribution. Also shown in the top panel are predicted offset distributions for NS-NS binary mergers in Milky Way type galaxies based on population synthesis models (Bloom et al. 1999; Fryer et al. 1999; Belczynski et al. 2006).

It lasted longer than and was softer than any previous burst, and was associated with SN 2006aj at only $z=0.033$. The BAT trigger enabled XRT and UVOT observations during the prompt phase of the GRB and initiated multiwavelength observations of the supernova from the time of the initial core collapse. The spectral peak in prompt emission at $\sim 5 \mathrm{keV}$ places GRB 060218 in the X-ray flash category of GRBs (Campana et al. 2006), the first such association for a GRB-SN event. Combined BAT-XRT-UVOT observations provided the first direct observation of shock-breakout in a SN (Campana et al. 2006). This is inferred from the evolution of a soft thermal component in the X-ray and UV spectra, and early time luminosity variations. Concerning the supernova, SN 2006aj was dimmer by a factor $\sim 2$ than the previous SNe associated with GRBs, but still $\sim 2-3$ times brighter than 
normal SN Ic not associated with GRBs (Pian et al. 2006; Mazzali et al. 2006). GRB 060218 was an underluminous burst, as were two of the other three previous cases. Because of the low luminosity, these events are only detected when nearby and are therefore rare occurrences. However, they are actually $\sim 5-10$ times more common in the universe than normal GRBs (Soderberg et al. 2006).

\section{Conclusions}

The future is bright for GRB astronomy. Swift will be in orbit for $>10$ more years and should be operating for at least 5 more years. To date, Swift has already detected more than $700 \mathrm{GRBs}$, and new things are constantly popping up. Swift has found that short GRBs reside in demonstrably different environments than long GRBs do, and are not accompanied by SNe. The spatial distributions over their host galaxies are much more spread out than for long GRBs (Fig. 6 - taken from Fong et al. 2010). The weight of the evidence lends credence to the NS-NS merger model. Distant GRBs are elucidating the properties of the high $z$ universe and probing into the era of re-ionization. In spite of the rich progress in the field, several open questions remain: What are the detailed properties of short GRBs? Do GRBs accompany the demise of the earliest stars? Is there direct evidence for beaming, as was once thought from panchromatic observations? How prominent a role do off-axis GRBs play?

\section{References}

Barthelmy, S.D., Barbier, L.M., Cummings, J.R., et al., 2005a, Space Sci. Rev., 120, 143

Barthelmy, S.D., Chincarini, G., Burrows, D.N., et al., 2005b, Nature, 438, 994

Belczynski, K., Perna, R., Bulik, T., et al., 2006, ApJ, 648, 1110

Berger, E., Price, P.A., Cenko, S.B., et al., 2005, Nature, 438, 988

Bloom, J.S., Prochaska, J.X., Pooley, D., et al., 2006, ApJ, 638, 354

Bloom, J.S., Sigurdsson, S., \& Pols, O.R., 1999, MNRAS, 305, 763

Burrows, D.N., Hill, J.E., Nousek, J.A., et al., 2005, Space Sci. Rev., 120, 165

Campana, S., Mangano, V., Blustin, A.J., et al., 2006, Nature, 442, 1008

Cenko, S.B., Fox, D.B., Penprase, B.E., et al., 2008, ApJ, 677, 441

Chandra, P., Cenko, S.B., Frail, D.A., et al., 2008, ApJ, 683, 924

Costa, E., Frontera, F., Heise, J., et al., 1997, Nature, 387, 783

Cucchiara, A., Levan, A.J., Fox, D.B., et al., 2011, ApJ, 736, 7

Evans, P.A., Beardmore, A.P., Page, K.L., et al., 2009, MNRAS, 397, 1177

Fong, W., Berger, E., \& Fox, D.B., 2010, ApJ, 708, 9

Fox, D.B., Frail, D.A., Price, P.A., et al., 2005, Nature, 437, 845

Frail, D.A., Kulkarni, S.R., Nicastro, L., et al., 1997, Nature, 389, 261

Fryer, C.L., Woosley, S.E., \& Hartmann, D.H., 1999, ApJ, 526, 152

Gehrels, N., Chincarini, G., Giommi, P., et al., 2004, ApJ, 611, 1005

Gehrels, N., Sarazin, C.L., O'Brien, P.T., et al., 2005, Nature, 437, 851 
Hjorth, J., Watson, D., Fynbo, J.P.U., et al., 2005, Nature, 437, 859

Klebesadel, R.W., Strong, I.B., \& Olson, R.A., 1973, ApJ, 182, L85

Kouveliotou, C., Meegan, C.A., Fishman, G.J., et al., 1993, ApJ, 413, L101

MacFadyen, A.I., \& Woosley, S.E., 1999, ApJ, 524, 262

Mazzali, P.A., Deng, J., Nomoto, K., et al., 2006, Nature, 442, 1018

Meegan, C.A., Fishman, G.J., Wilson, R.B., et al., 1991, Nature, 355, 143

Onda, K., Tashiro, M.S., Nakagawa, Y.E., et al., 2010, PASJ, 62, 547

Pian, E., Mazzali, P.A., Masetti, N., et al., 2006, Nature, 442, 1011

Roming, P.W.A., Kennedy, T.E., Mason, K.O., et al., 2005, Space Sci. Rev., 120, 95

Sakamoto, T., Barthelmy, S.D., Barbier, L., et al., 2008, ApJS, 175, 179

Sakamoto, T., Barthelmy, S., Baumgartner, W., et al., 2011, in Gamma Ray Bursts 2010, ed. J.E. McEnery, J.L. Racusin \& N. Gehrels (AIP: New York), 1358, 51

Soderberg, A.M., Kulkarni, S.R., Nakar, E., et al., 2006, Nature, 442, 1014

van Paradijs, J., Groot, P.J., Galama, T., et al., 1997, Nature, 386, 686

Villasenor, J.S., Lamb, D.Q., Ricker, G.R., et al., 2005, Nature, 437, 855 
